\title{
Spontaneous movements and linear response of a noisy oscillator
}

\author{
F. Jülicher ${ }^{1}$, K. Dierkes ${ }^{1}$, B. Lindner ${ }^{1}$, a, J. Prost ${ }^{2}$, and P. Martin ${ }^{2}$ \\ 1 Max Planck Institut für Physik komplexer Systeme, Dresden, Germany \\ 2 Physico-chimie Curie, CNRS, Institut Curie, UPMC, Paris, France
}

Received 3 March 2009 and Received in final form 26 May 2009

Published online: 23 August 2009 - (c) EDP Sciences / Società Italiana di Fisica / Springer-Verlag 2009

\begin{abstract}
A deterministic system that operates in the vicinity of a Hopf bifurcation can be described by a single equation of a complex variable, called the normal form. Proximity to the bifurcation ensures that on the stable side of the bifurcation (i.e. on the side where a stable fixed point exists), the linear-response function of the system is peaked at the frequency that is characteristic of the oscillatory instability. Fluctuations, which are present in many systems, conceal the Hopf bifurcation and lead to noisy oscillations. Spontaneous hair bundle oscillations by sensory hair cells from the vertebrate ear provide an instructive example of such noisy oscillations. By starting from a simplified description of hair bundle motility based on two degrees of freedom, we discuss the interplay of nonlinearity and noise in the supercritical Hopf normal form. Specifically, we show here that the linear-response function obeys the same functional form as for the noiseless system on the stable side of the bifurcation but with effective, renormalized parameters. Moreover, we demonstrate in specific cases how to relate analytically the parameters of the normal form with added noise to effective parameters. The latter parameters can be measured experimentally in the power spectrum of spontaneous activity and linear-response function to external stimuli. In other cases, numerical solutions were used to determine the effects of noise and nonlinearities on these effective parameters. Finally, we relate our results to experimentally observed spontaneous hair bundle oscillations and responses to periodic stimuli.
\end{abstract}

PACS. 43.64.Bt Models and theories of the auditory system - 82.40.Bj Oscillations, chaos, and bifurcations - 05.10.Gg Stochastic analysis methods (Fokker-Planck, Langevin, etc.)

\section{Introduction}

A wide range of complex systems, including lasers, chemical reactions, electronic circuits, biological cells, and neural networks, display self-sustained oscillations. Generally, these oscillators are subjected to intrinsic or external noise and often play a role as active subunits within a larger system. The properties of such oscillators can be characterized by their spontaneous activity and their response to external perturbations. Since such oscillators are governed by nonlinear dynamics, only a limited number of analytical results characterizing the spontaneous activity and the response are known.

The behavior of a complex system is characterized by a large number of coupled degrees of freedom. A deterministic description of any nonlinear system is greatly simplified, however, if this system operates in the vicinity of an oscillatory instability, the Hopf bifurcation. First, the relaxation dynamics as well as the response to sinusoidal stimuli with frequencies close to the characteristic frequency of the oscillatory instability are governed by

\footnotetext{
a e-mail: benji@pks.mpg.de
}

only two degrees of freedom. Furthermore, through a sequence of analytic, but nonlinear, coordinate changes, the equations describing the dynamics of these two degrees of freedom can be condensed into a single equation, called the normal form, of a single complex variable $z$ [1]. This nonlinear transformation separates the generic dynamical part of the nonlinearities (surviving in the Hopf normal form) from system-specific nonlinearities (included in the nonlinear transformation). By adding noise to this normal form, one can describe the spontaneous activity of a noisy oscillator $[2-6]$ as well as the response to a periodic driving. With a stochastic driving included in the dynamics, it is not possible to talk about bifurcation points anymore - the term bifurcation region coined by Meunier and Verga [7] is more appropriate. One could also say that the sharp bifurcation is concealed, which leads on the stable side to noisy precursors of the bifurcation [8], but also has consequences on the oscillatory side as we will see in this paper.

Generally, self-sustained oscillators are ideally suited to detect and amplify weak signals near a characteristic frequency. It has been suggested that this principle is 
employed in the ear of vertebrates [9-11], reviewed in [12]. The mechanosensory hair cells in the inner ear are each endowed with a mechanical antenna, the hair bundle, that can oscillate spontaneously [13-19]. It has been shown experimentally for hair cells from the bullfrog that a hair bundle's responsiveness to sinusoidal stimuli is tuned to the characteristic frequency of spontaneous oscillations and displays a domain of compressive nonlinearity when driven with stimuli of increasing magnitudes [20]. These properties have been recognized as signatures of a dynamical system that operates close to a Hopf bifurcation. In addition, hair bundle oscillations are rather noisy. In the presence of noise, the Hopf bifurcation is concealed and the sensitivity that the system can achieve in response to small stimuli is limited [21].

In general, we cannot infer the parameters of the normal form describing an oscillator from noisy physical observables. Put differently, in a physical system (such as the hair bundle), the physical observables (e.g. the displacement of the hair bundle) do not coincide with the variables of the normal form and do not represent all relevant degrees of freedom. Even if we can determine solutions for the normal form, it is unclear how to relate them to measurements of these physical observables. Another important issue is whether such measurements, as for instance, that of a hair bundle displacement, are sufficient to completely determine the parameters of the normal form even if the physical observables do not comprise the complete set of dynamical variables.

Here, we start with a simple model that captures the linear mechanical behavior of an oscillatory hair bundle with two degrees of freedom [18]. We then add nonlinearities and analyze the behavior of a noisy nonlinear oscillator in several steps. In sect. 2, we derive the normal form of the oscillator in the absence of noise. In sect. 3 we include noise in the description and solve numerically the time-dependent Fokker-Planck equation for the probability density of the normal form variable. We also give approximate analytical solutions for limiting cases. In sect. 4 we show that in the limit of a low intrinsic noise, the linear response of the noisy system obeys the same functional form as a deterministic dynamical system operating on the stable side of a supercritical Hopf bifurcation, however, with renormalized parameters (here and in the following we refer to the side of the bifurcation where a stable fixed point exists as the stable side of the bifurcation). We use the analytical results from sect. 3 to calculate these effective parameters as functions of bare parameters of the normal form. This allows us to give expressions for the power spectrum and the response function that very well fit numerical simulations. In sect. 5 we relate the theory to the properties of sensory hair bundles from the inner ear.

This work is complementary to a recent study by some of the authors in which the stochastic hair bundle dynamics was studied in another simplificiation, namely a twostate description [22]. While the latter approach is justified for a system operating deep in the oscillatory regime and may capture certain aspects of the hair-bundle dynamics more faithfully (say, the relaxation oscillations), our ap- proach here is more general and applies to a variety of systems operating near a supercritical Hopf bifurcation.

\section{Deterministic description of an active oscillator}

In this section, we introduce the two relevant degrees of freedom for an oscillatory hair bundle and derive the normal form of the oscillator. We start by writing an equation to describe the linear behavior of the hair bundle deflection $X[18]$

$$
\lambda \frac{\mathrm{d} X}{\mathrm{~d} t}=-k X+F_{a}+F_{\text {ext }} .
$$

Here, $\lambda$ and $k$ are, respectively, the drag coefficient and the stiffness of the hair bundle. The force $F_{a}$ is a force generated by active elements within the hair bundle such as motors or ion channels. The system is stimulated by a periodic external force $F_{\text {ext }}$. For the system to oscillate spontaneously, the active force $F_{a}$ must provide positive feedback to $X$. We write to linear order

$$
\beta \frac{\mathrm{d} F_{a}}{\mathrm{~d} t}=-F_{a}-\bar{k} X
$$

Here, $\beta$ is a relaxation rate of the active process and the coupling coefficient $\bar{k}$ has dimensions of a spring constant. Note that $X$ and $F_{a}$ represent the two relevant degrees of freedom of the oscillator. The combined equations (1) and (2) describe the linear behavior of an active system and cannot be derived from a potential.

\subsection{Linear equations}

In order to discuss the linear equations, it is useful to perform a coordinate change. This procedure is described in appendix A. In short, we first write eqs. (1) and (2) in matrix form

$$
\dot{x}_{i}=A_{i j} x_{j}+f_{i},
$$

where $i=x, a$ and we use the notation $x_{x}=X, x_{a}=F_{a}$ and $f_{x}=F_{\text {ext }} / \lambda$. Because the system is oscillatory, the matrix displays two complex-conjugate eigenvalues which we denote as $-r-i \omega_{0}$ and $-r+i \omega_{0}$. We diagonalize the matrix by using a transformation matrix $\mathbf{M}$, thereby defining the complex variables

$$
z_{i}=M_{i j}^{-1} x_{j},
$$

with $z=z_{x}=z_{a}^{*}$. The system can thus be described by the single complex equation

$$
\dot{z}=-\left(r+i \omega_{0}\right) z+f
$$

where we have defined the complex force $f=M_{x j}^{-1} f_{j}$. We choose the coordinate change such as to write

$$
X=\frac{z+z^{*}}{2} \text {. }
$$


The relation between the external force $F_{\text {ext }}$ and the complex force $f$ is given by

$$
f=\frac{e^{-i \alpha}}{\Lambda} F_{\text {ext }}
$$

where $\Lambda$ and $\alpha$ are, respectively, the amplitude and phase of

$$
\Lambda e^{i \alpha}=\lambda\left(1-\frac{i}{2 \omega_{0}}\left(\frac{k}{\lambda}-\frac{1}{\beta}\right)\right)^{-1} \text {. }
$$

\subsection{Linear-response function}

As long as $r>0$, the oscillator is stable and, for small external forces, we can ignore nonlinearities. We are interested in the properties of the linear-response function $\chi_{X F}$, defined as

$$
X(t) \simeq \int_{-\infty}^{t} \mathrm{~d} t^{\prime} \chi_{X F}\left(t-t^{\prime}\right) F_{\mathrm{ext}}\left(t^{\prime}\right)
$$

This response function can be calculated from the response function $\chi_{z F}$ that relates the stimulus force $F_{\text {ext }}$ and $z$. In Fourier representation $\tilde{z}(\omega) \simeq \tilde{\chi}_{z F} \tilde{F}_{\text {ext }}(\omega)$, with

$$
\tilde{\chi}_{z F}(\omega)=\frac{e^{-i \alpha}}{i \Lambda\left(\omega_{0}-\omega\right)+K}
$$

where we have introduced the stiffness $K=\Lambda r>0$. The response function $\tilde{\chi}_{X F}(\omega)$ for the Fourier mode $\tilde{X}(\omega)=$ $\left(\tilde{z}(\omega)+\tilde{z}^{*}(-\omega)\right) / 2$ can be expressed as

$$
\tilde{\chi}_{X F}(\omega)=\frac{1}{2}\left(\frac{e^{-i \alpha}}{i \Lambda\left(\omega_{0}-\omega\right)+K}+\frac{e^{+i \alpha}}{-i \Lambda\left(\omega_{0}+\omega\right)+K}\right) .
$$

Note that, for $\omega \simeq \omega_{0}$ and $\omega_{0} \gg r$,

$$
\tilde{\chi}_{X F}^{-1}(\omega) \simeq 2 \tilde{\chi}_{z F}^{-1}(\omega)
$$

In time domain,

$$
\chi_{X F}(t)=\theta(t) \frac{e^{-(K / \Lambda) t}}{\Lambda} \cos \left(\omega_{0} t+\alpha\right),
$$

where the Heavyside function, $\theta(t)=1$ for $t>0$ and $\theta=0$ otherwise, ensures causality.

As $r$ vanishes or becomes negative, there is no linear response of the deterministic system at its characteristic frequency. This is consistent with the divergence of the linear-response function if $K \rightarrow 0$ (i.e. for $r \rightarrow 0$ ).

\subsection{Nonlinearities and normal form of the oscillator}

The linear coordinate change given by eq. (4) permits a description of our two-dimensional system (1) and (2) by a single equation (5) of a complex variable $z$. This equation represents the normal form of the oscillator to linear order. In general, the dynamic equations of the system will also contain nonlinearities, which become important at the Hopf bifurcation.

Near the bifurcation, nonlinear terms can be brought into normal form by adding appropriate nonlinear corrections to the variable $z: z_{i}=M_{i j}^{-1} x_{j}+O\left(x_{i} x_{j}\right)$ (see chapt. 2.2 in ref. [1]). Note that the linear coordinate change (4) does not affect the structure of nonlinear terms. The normal form is characterized by the condition of phase invariance $z \rightarrow z e^{i \phi}$, not only for the linear term, but for all nonlinearities. This condition excludes quadratic nonlinearities and imposes a cubic nonlinearity of the form $|z|^{2} z$ yielding the normal-form dynamics

$$
\frac{\mathrm{d} z}{\mathrm{~d} t}=-\left(r+i \omega_{0}\right) z-B\left|z^{2}\right| z+O\left(\left|z^{4}\right| z\right)+f
$$

where we have introduced a complex coefficient $B=(b+$ $\left.i b^{\prime}\right)$.

The normal form (14) describes the generic dynamics of the variable $z$. However, this form is of interest only if it provides insights into the behavior of the physical variables $X$ and $F_{a}$. A nonlinear system stimulated by a sinusoidal periodic force $F(t)=F_{1} e^{-i \omega t}+F_{-1} e^{i \omega t}$ responds with all higher harmonics, i.e. $X(t)=\sum_{n} X_{n} e^{-i n \omega t}$. It can be shown on general grounds (see supplementary material in ref. [10]) that, for small $F_{1}$, the first mode $X_{1}$ dominates and can be expanded as

$$
F_{1}=A_{X F} X_{1}+B_{X F}\left|X_{1}^{2}\right| X_{1}+O\left(\left|X_{1}^{4}\right| X_{1}\right) .
$$

In appendix $\mathrm{B}$, we demonstrate that the coefficients $A_{X F}$ and $B_{X F}$ are directly related to the coefficients introduced in the normal form:

$$
\begin{aligned}
& A_{X F} \simeq 2 \Lambda e^{i \alpha}\left(i\left(\omega_{0}-\omega\right)+r\right), \\
& B_{X F} \simeq 8 \Lambda e^{i \alpha} B .
\end{aligned}
$$

\subsection{Hopf bifurcation}

If $r>0$, the system is quiescent and $X_{1}=0$. If the parameter $r$ changes sign and becomes negative, the system undergoes a Hopf bifurcation and has limit-cycle solutions. In the absence of an external force, $F_{1}=0$, eq. (15) displays nontrivial solutions corresponding to limit cycles with amplitude

$$
\left|X_{1}\right|^{2}=-A_{X F} / B_{X F} .
$$

A spontaneous oscillation can only exist at a particular frequency $\omega=\omega_{c}=\omega_{0}+\left(b^{\prime} / b\right)|r|$ for which $A_{X F} / B_{X F}$ is a real negative number. In this case, the system oscillates at the frequency $\omega_{c}$ with an amplitude

$$
\left|X_{1}\right|=\frac{1}{2}\left|\frac{r}{b}\right|^{1 / 2}
$$

This is the classic scenario of a Hopf bifurcation. At the critical point, $r$ vanishes. For $\omega=\omega_{0}$, the response is essentially nonlinear with $\left|X_{1}\right| \sim F_{1}^{1 / 3}$ : no matter how small the external force, the response is nonlinear. If instead $\delta \omega=\omega_{0}-\omega$ is finite, there always remains a linear regime for small forces. 


\section{Effects of fluctuations - Noisy oscillations}

We now discuss the situation in which noise affects the system. In this case, the normal form (14) becomes a stochastic differential equation. Fluctuations are described by a noise term $\xi$, which in general does not satisfy phase invariance. If fluctuations are weak, however, the phase-invariant component of the noise dominates the system (see appendix C). A simple choice is given by Gaussian white noise with $\langle\xi\rangle=0,\left\langle\xi(t) \xi\left(t^{\prime}\right)\right\rangle=0$ and $\left\langle\xi(t) \xi^{*}\left(t^{\prime}\right)\right\rangle=4 d \delta\left(t-t^{\prime}\right)$, where we have excluded the phase-dependent component $\left\langle\xi(t) \xi\left(t^{\prime}\right)\right\rangle$ (here and in the following $\langle\cdots\rangle$ denotes the average over the white noise).

We thus arrive at the normal form of a noisy oscillator

$$
\dot{z}=-\left(r+i \omega_{0}\right) z-\left(b+i b^{\prime}\right)|z|^{2} z+\bar{f} e^{-i \omega t}+\xi .
$$

In the presence of the external periodic force $f=f_{1} e^{-i \omega t}$, we can choose $f_{1}=\bar{f}$ real without loss of generality. For numerical evaluations, we have used a dimensionless expression of the normal form, thereby reducing the number of parameters by three (see appendix D).

\subsection{Statistical measures of spontaneous and driven movements}

We are interested in the time-dependent average $\langle z(t)\rangle$ and in the correlation function $\left\langle z(t) z^{*}\left(t^{\prime}\right)\right\rangle$. The latter is related to the spectral density

$$
\begin{aligned}
\tilde{S}(\omega)= & \int_{-T / 2}^{T / 2} \frac{\mathrm{d} t}{T} \int_{-T / 2}^{T / 2} \mathrm{~d} t^{\prime}\left\langle z(t) z^{*}\left(t^{\prime}\right)\right\rangle e^{i \omega\left(t-t^{\prime}\right)} \\
= & \int_{-T / 2}^{T / 2} \frac{\mathrm{d} t}{T} \int_{-T / 2}^{T / 2} \mathrm{~d} t^{\prime}\left[\left\langle z(t) z^{*}\left(t^{\prime}\right)\right\rangle_{c}\right. \\
& \left.+\langle z(t)\rangle\left\langle z^{*}\left(t^{\prime}\right)\right\rangle\right] e^{i \omega\left(t-t^{\prime}\right)},
\end{aligned}
$$

where $T$ is the time interval of observation and $\left\langle z z^{*}\right\rangle_{c}=$ $\left\langle(z-\langle z\rangle)\left(z^{*}-\left\langle z^{*}\right\rangle\right)\right\rangle$ is the connected autocorrelation function. In the presence of an external stimulus $f=f_{1} e^{-i \omega_{1} t}$, the average $\langle z(t)\rangle=z_{1} e^{-i \omega_{1} t}$ is nonzero and consequently there exists a Fourier mode with

$$
\langle\tilde{z}(\omega)\rangle=2 \pi z_{1} \delta\left(\omega-\omega_{1}\right) .
$$

For a weak driving, $z_{1}$ determines the linear-response function as $\chi_{z f}=\mathrm{d} z_{1} / \mathrm{d} f_{1}$ for sufficiently small $f_{1}$. Furthermore,

$$
\tilde{S}(\omega)=\tilde{S}_{0}(\omega)+2 \pi\left|z_{1}\right|^{2} \delta\left(\omega-\omega_{1}\right),
$$

where $\tilde{S}_{0}(\omega)$ is the Fourier transform of the connected correlation function $S_{0}(t)=\left\langle z(t) z^{*}(0)\right\rangle_{c}$. In the absence of the stimulus, $\langle z(t)\rangle=0$ and $S=S_{0}$.

\subsection{Fokker-Planck equation and linear response of the noisy oscillator}

In order to calculate averages and correlation functions, we write a Fokker-Planck equation for the probability $P(z, t)$ to find the system at $z$ in the complex plane at time $t$ (for the derivation of the Fokker-Planck equation from the Langevin equation, see [4]). For simplicity, we use the Gaussian white noise introduced above with $\left\langle\xi(t) \xi^{*}\left(t^{\prime}\right)\right\rangle=$ $4 d \delta\left(t-t^{\prime}\right)$.

Starting with the Langevin equation (20), we obtain the corresponding Fokker-Planck equation for the distribution $P(\rho, \phi, t)$, where points in the complex plane are represented by polar coordinates $z=\rho e^{i \phi}$ :

$$
\begin{aligned}
& \partial_{t} P=\partial_{\rho}\left[\left(r \rho+b \rho^{3}-\bar{f} \cos (\phi+\omega t)-\frac{d}{\rho}\right) P+d \partial_{\rho} P\right] \\
& +\partial_{\phi}\left[\left(\omega_{0}+b^{\prime} \rho^{2}+\frac{\bar{f}}{\rho} \sin (\phi+\omega t)\right) P+\frac{d}{\rho^{2}} \partial_{\phi} P\right] .
\end{aligned}
$$

This distribution satisfies the normalization condition $\int P(\rho, \phi) \mathrm{d} \rho \mathrm{d} \phi=1$. In the absence of an external force, $\bar{f}=0$, this equation has the steady-state solution $P_{s}=$ $N \rho \exp \{-W / d\}$ with $\partial_{t} P_{s}=0$ and

$$
W=\frac{1}{2} r \rho^{2}+\frac{b}{4} \rho^{4} .
$$

The linear response of the system can be discussed by writing

$$
P(\rho, \phi, t) \simeq P_{s}\left(1+P_{1}\right) .
$$

To linear order in $\bar{f}, P_{1}$ satisfies the equation

$$
\begin{aligned}
\partial_{t} P_{1}= & \frac{d}{\rho} \partial_{\rho} P_{1}+d \partial_{\rho}^{2} P_{1}-\frac{\mathrm{d} W}{\mathrm{~d} \rho} \partial_{\rho} P_{1}+\left(b^{\prime} \rho^{2}+\omega_{0}\right) \partial_{\phi} P_{1} \\
& +\frac{d}{\rho^{2}} \partial_{\phi}^{2} P_{1}+\frac{\bar{f} \cos (\phi+\omega t)}{d} \frac{\mathrm{d} W}{\mathrm{~d} \rho}
\end{aligned}
$$

This equation is solved by the Ansatz

$$
P_{1}=Q(\rho, \omega) e^{-i(\phi+\omega t)}+Q^{*}(\rho, \omega) e^{i(\phi+\omega t)},
$$

where $Q$ satisfies

$-d \frac{\mathrm{d}^{2}}{\mathrm{~d} \rho^{2}} Q+\frac{\mathrm{d} W}{\mathrm{~d} \rho} \frac{\mathrm{d}}{\mathrm{d} \rho} Q+i\left(\delta \omega+b^{\prime} \rho^{2}\right) Q=d \frac{\mathrm{d}}{\mathrm{d} \rho}\left(\frac{Q}{\rho}\right)+\frac{\bar{f}}{2 d} \frac{\mathrm{d} W}{\mathrm{~d} \rho}$.

A similar equation has been discussed by several authors for the related problem of calculating the spontaneous power spectrum of the noisy normal form $[2,6]$. Note that the function $Q$ depends on the frequency only via the detuning $\delta \omega=\omega_{0}-\omega$ between driving and eigenfrequency. Constraints on the probability $P(\rho, \phi, t)$ provide boundary conditions for eq. (30). First, because $\rho \geq 0$, the probability flux $J_{\rho}(\rho, \phi, t)$ must vanish at all times and phases at the point $\rho=0$ :

$$
\begin{aligned}
& J_{\rho}(\rho=0, \phi, t)=\left(\frac{d}{\rho}+\bar{f} \cos (\phi+\omega t)-\frac{\mathrm{d} W}{\mathrm{~d} \rho}-d \frac{\mathrm{d}}{\mathrm{d} \rho}\right) P_{s} \\
& \times\left.\left[1+Q e^{-i(\phi+\omega t)}+Q^{*} e^{i(\phi+\omega t)}\right]\right|_{\rho=0}=0 .
\end{aligned}
$$

From this, we get that $\lim _{\rho \rightarrow 0} \rho \partial_{\rho} Q(\rho)=0$, which excludes divergences of $|Q|$ at $\rho=0$ by power laws $\rho^{-\alpha}$ 
(with $\alpha>0$ ) or by a logarithmic divergence. Near $\rho=0$, we can thus use a power series expansion of $Q(\rho)$

$$
Q(\rho)=\sum_{k=0} C_{k} \rho^{k}
$$

Inserting this expansion into eq. (30) yields exactly one diverging term $C_{0} / \rho^{2}$. We thus impose $C_{0}=0$ and get the first boundary condition

$$
Q(0)=0
$$

Second, for large $\rho$, the solution for $Q$ which describes linear response is proportional to $\rho$, which yields

$$
\left.Q(\rho)\right|_{\rho \rightarrow \infty}=\frac{\bar{f}}{2 d} \frac{b}{b+i b^{\prime}} \rho .
$$

We note that if both $b$ and $b^{\prime}$ are exactly zero,

$$
\left.Q(\rho)\right|_{\rho \rightarrow \infty}=\frac{\bar{f}}{2 d} \frac{r}{i\left(\omega_{0}-\omega\right)+r} \rho .
$$

Equations (33) and (34) are boundary conditions for eq. (30) which ensure that $Q$ vanishes for $\bar{f}=0$.

Equation (30) can be solved in two special cases: i) $b=$ $b^{\prime}=0, r>0$ (the linear stable case) where it is solved by the right-hand side of eq. (35); ii) $b^{\prime}=0, \delta \omega=0$ (no detuning between driving frequency and eigenfrequency $\left.\omega_{0}\right)$ where $Q$ becomes a simple linear function in $\rho$

$$
Q=\frac{\bar{f}}{2 d} \rho \quad \text { for } \quad b^{\prime}=0, \delta \omega=0 .
$$

In general eq. (30) cannot be solved analytically but can be integrated numerically using the boundary conditions provided by eqs. (33) and (34), the former condition being used at small but finite $\rho=\epsilon$ to avoid the singularity at $\rho=0$. Numerical solutions of the Fokker-Planck eq. (30) were compared in two cases to simulation results of the Langevin equation (20) in polar coordinates; we found excellent agreement between these two approaches (figs. 1 and 2). The first case is the analytically solvable case $b^{\prime}=0$ and $\delta \omega=0$ yielding a purely real and linearly growing function $Q(\rho)$ shown in fig. 1. The total modulation of the probability density is quite strong for a modest driving amplitude of $\bar{f}=0.1$. The second case shown in fig. 2 is far away from these conditions: here $b^{\prime}=1$ and $\delta \omega=0.5$. Typically, for a signal slower than the eigenfrequency of the system, the phase shift (the complex phase of $Q$, cf. fig. 2B) remains negative for all $\rho$. The absolute value $|Q|$ can exhibit a local minimum around the minimum of the potential $W(\rho)$ (for the numerical example in fig. $2 \mathrm{C}$ around $\rho=1$ ), and the total modulation of the probability density is weak because the system is driven off resonance. Data for $b^{\prime}=0$ and $\delta \omega=0.5$ look similar except that the modulus shows only a mild nonlinear growth with increasing $\rho$ instead of a minimum (not shown).

For $b^{\prime}=0$, it is still possible to obtain an analytical estimate for $Q$ for small detuning $\delta \omega \ll 1$. To this end,

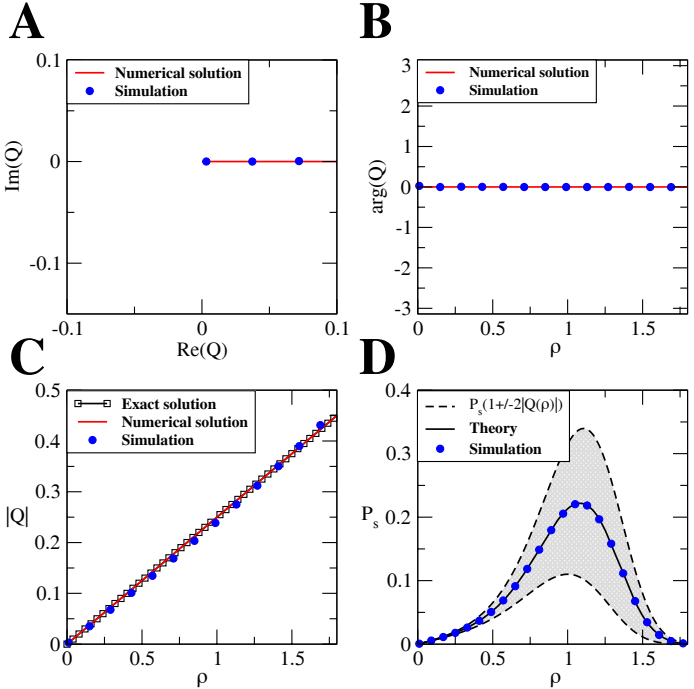

Fig. 1. (Colour on-line) Linear-response characteristics of the probability density $Q$ to periodic driving in the solvable case $b^{\prime}=0$ and $\omega=\omega_{0}=1$. Remaining parameters: $d=0.2, \bar{f}=$ $0.1, r=-1, b=1$. In these plots, the red line corresponds to a numerical integration of the differential equation (30) for $Q$ with the boundary conditions provided by eqs. (33) and (34). The solid line with squares shown in panel $\mathrm{C}$ corresponds to the exact solution (eq. (36)). The blue circles have been extracted from stochastic simulations of the Langevin equations (20) in polar coordinates $(\rho, \phi)$ (using a simple Euler scheme with a dynamical time step smaller than $4 \times 10^{-4}$ ). In these simulations, by averaging over time (about $15 \times 10^{6}$ periods of the driving) we have measured the density of the driven system as seen in a coordinate system that is co-rotating with the signal. For fixed $\rho$ the first Fourier coefficient with respect to the phase $\phi$ then yields, according to eq. (29), the function $P_{s}(\rho) Q(\rho)$. From this product we can estimate $Q(\rho)$ using for consistency $P_{s}(\rho)$ as determined from simulations in the absence of periodic drive. The striking agreement, here and in other simulations, between the numerical solution of eq. (30) and the result of Langevin simulations validates the linear-response theory at the driving magnitude $\bar{f}=0.1$.

a first-order expansion with respect to $\delta \omega$ and a secondorder expansion with respect to $\rho$ is sufficient and yields (see appendix E)

$$
Q(\rho)=\frac{\bar{f}}{2 d}\left[\rho+i \delta \omega\left(a_{1} \rho+a_{2} \rho^{2}\right)\right],
$$

where the coefficients are given by

$$
\begin{aligned}
& a_{1}=\frac{1}{d} \frac{b\left\langle\rho^{5}\right\rangle\langle\rho\rangle-d\left\langle\rho^{2}\right\rangle+r\left\langle\rho^{3}\right\rangle\langle\rho\rangle}{b\left\langle\rho^{4}\right\rangle+r\left\langle\rho^{2}\right\rangle-b\left\langle\rho^{-1}\right\rangle\left\langle\rho^{5}\right\rangle-r\left\langle\rho^{-1}\right\rangle\left\langle\rho^{3}\right\rangle}, \\
& a_{2}=-\frac{1}{d} \frac{-d\left\langle\rho^{-1}\right\rangle\left\langle\rho^{2}\right\rangle+b\langle\rho\rangle\left\langle\rho^{4}\right\rangle+r\langle\rho\rangle\left\langle\rho^{2}\right\rangle}{b\left\langle\rho^{4}\right\rangle+r\left\langle\rho^{2}\right\rangle-b\left\langle\rho^{-1}\right\rangle\left\langle\rho^{5}\right\rangle-r\left\langle\rho^{-1}\right\rangle\left\langle\rho^{3}\right\rangle} .
\end{aligned}
$$

Here $\langle\ldots\rangle$ denotes an average with respect to the stationary distribution $P_{s}$. 


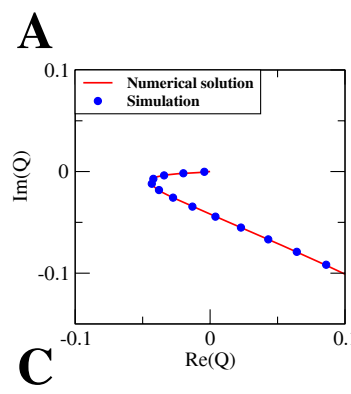

B
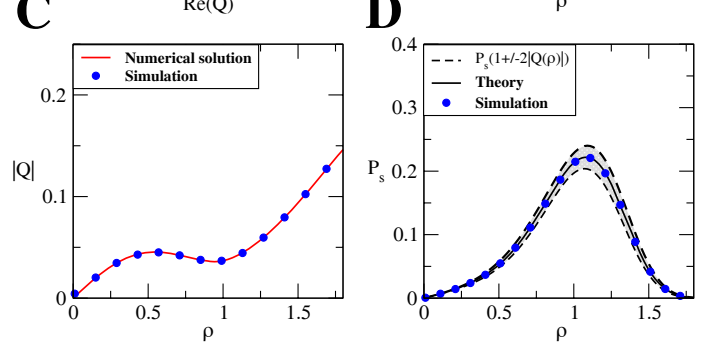

Fig. 2. Linear-response characteristics of the probability density $Q$ to periodic driving. Here $b^{\prime}=1, \omega=0.5, \omega_{0}=1$; other parameters and symbols as in fig. 1.
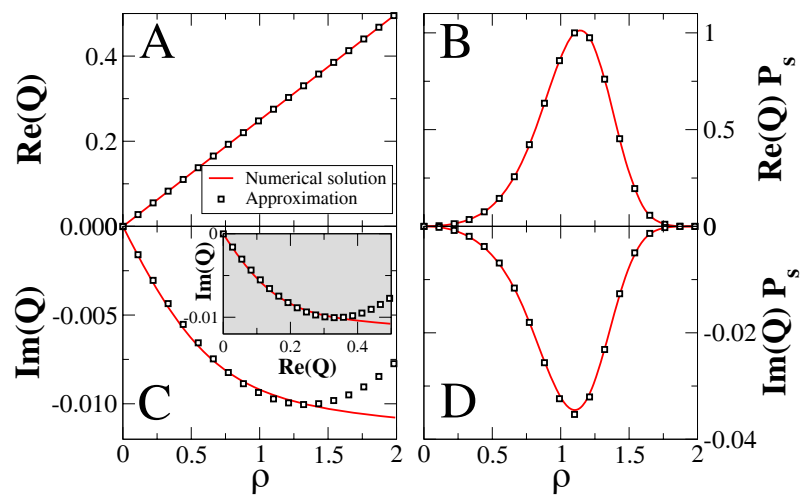

Fig. 3. Approximation eq. (37) and exact numerical solution for the function $Q$ (A and $\mathrm{C}$ ) and the function $Q P_{s}(\rho)$ for a small detuning $\delta \omega=0.01$; other parameters as in fig. 1. Shown are the real parts (A and B) and imaginary parts $(\mathrm{C}$ and $\mathrm{D})$ of the two functions. The inset in $\mathrm{C}$ displays imaginary against real part of $Q$ with $\rho$ as a parameter - this should be compared to panels A in figs. 1 and 2 and thus reveals how the transition from figs. $1 \mathrm{~A}$ to $2 \mathrm{~A}$ with growing detuning takes place.

Knowing $Q$, the value of $z_{1}$ in linear response can be calculated as

$$
z_{1}=\langle\rho Q(\rho)\rangle=\int_{0}^{\infty} \mathrm{d} \rho \rho Q(\rho) P_{s}(\rho) .
$$

Note that this equation can be regarded also as an average of $\rho$ with respect to the function $Q(\rho) P_{s}(\rho)$, the latter being the additive modification of the probability density caused by the periodic signal.

In fig. 3 we show the functions $Q(\rho)$ and $Q(\rho) P_{s}(\rho)$ (real and imaginary parts) in the case of $b^{\prime}=0$ and a small detuning $(\delta \omega=0.01)$ together with the approximation eq. (37) (empty squares). The real parts are well approximated by the function at resonance (i.e. the exact solution for $\delta \omega=0$ given in eq. (36)). The imaginary part of the approximation eq. (37) shows good agreement for a range of small to moderate values of $\rho$, deviates strongly, however, for larger $\rho$ (cf. fig. 3C); in particular, the solution eq. (37) cannot and does not obey the boundary condition at large $\rho$, eq. (34). The approximation for the product $Q(\rho) P_{s}(\rho)$ agrees, however, very well with the true function (cf. fig. 3D) since the values at large $\rho$ are exponentially damped by the stationary density. We thus expect that eq. (37) will give a reasonable agreement for calculating the linear response via eq. (40) for small detuning.

\section{Effective parameters characterizing noisy oscillations and their relation to the normal form}

\subsection{Phenomenological description of spectral measures}

In the presence of noise the parameters $K, \Lambda, \omega_{0}, \alpha$ and $B$ are renormalized. We can define effective parameters by first looking at the linear-response function of the noisy nonlinear system $\tilde{\chi}_{z F}(\omega)=\mathrm{d} z_{1} / \mathrm{d} F_{1}=\Lambda^{-1} e^{-i \alpha} \mathrm{d} z_{1} / \mathrm{d} f_{1}$ for $f_{1}=0$. The inverse of $\mathrm{d} z_{1} / \mathrm{d} f_{1}$ at $f_{1}=0$ is a complex function

$$
\frac{\mathrm{d} f_{1}}{\mathrm{~d} z_{1}}=G(\omega) e^{i \theta(\omega)}
$$

of $\omega$ with modulus $G$ and phase $\theta$. We define the effective frequency $\omega_{0}^{\text {eff }}$ as the frequency of maximal absolute response, i.e. at which $G$ is minimal and

$$
\left.\frac{\mathrm{d} G}{\mathrm{~d} \omega}\right|_{\omega=\omega_{0}^{\text {eff }}}=0 .
$$

We can now expand $G$ and $\phi$ at $\omega=\omega_{0}^{\text {eff }}$

$$
\begin{aligned}
G & \simeq G_{0}+G_{1}\left(\omega-\omega_{0}^{\mathrm{eff}}\right)^{2}, \\
\theta & \simeq \theta_{0}+\theta_{1}\left(\omega-\omega_{0}^{\mathrm{eff}}\right) .
\end{aligned}
$$

As a result, the inverse of the effective linear-response function $\tilde{\chi}_{z F}$ can be written to linear order in $\omega_{0}^{\text {eff }}-\omega$ as

$$
\tilde{\chi}_{z F}^{-1} \simeq \tilde{\chi}_{X F}^{-1} / 2 \simeq e^{i \alpha_{\mathrm{eff}}}\left(K_{\mathrm{eff}}+i \Lambda_{\mathrm{eff}}\left(\omega_{0}^{\mathrm{eff}}-\omega\right)\right) .
$$

Here, $K_{\text {eff }}=\Lambda G_{0}, \Lambda_{\text {eff }}=-\Lambda G_{0} \theta_{1}, \alpha_{\text {eff }}=\alpha+\theta_{0}$. Thus, the linear response function $\tilde{\chi}_{X F}$ has the same form as eq. (11) of the noiseless problem, but with effective, renormalized parameters. We note that the approximation given by eq. (45) is appropriate for systems with a sharply peaked response $\left(\omega_{0}^{\text {eff }} \gg K_{\text {eff }} / \Lambda_{\text {eff }}\right)$.

The autocorrelation function in the absence of a stimulus can also be discussed via its Fourier transform $\tilde{S}_{0}(\omega)$. We can define an effective noise strength $D_{\text {eff }}(\omega)$ by introducing an effective random force $\xi_{\text {eff }}$ with spectral density $\left\langle\tilde{\xi}_{\text {eff }}(\omega) \tilde{\xi}_{\text {eff }}^{*}(\omega)\right\rangle=4 D_{\text {eff }}$ and $\left\langle\tilde{\xi}_{\text {eff }}(\omega) \tilde{\xi}_{\text {eff }}(\omega)\right\rangle=0$ to accord with the phase-invariance condition. With $\tilde{z}(\omega) \simeq$ $\tilde{\chi}_{z F} \tilde{\xi}_{\text {eff }}(\omega)$, we write $\tilde{S}_{0}(\omega)=\left\langle\tilde{z}(\omega) \tilde{z}^{*}(\omega)\right\rangle \simeq 4\left|\tilde{\chi}_{z F}\right| D_{\text {eff }}$. Therefore

$$
\tilde{S}_{0}(\omega) \simeq \frac{4 D_{\mathrm{eff}}(\omega)}{K_{\mathrm{eff}}^{2}+\Lambda_{\mathrm{eff}}^{2}\left(\omega_{0}^{\mathrm{eff}}-\omega\right)^{2}}
$$


The spectral density of the spontaneous movement $\tilde{C}_{0}(\omega)=\langle\tilde{X}(\omega) \tilde{X}(-\omega)\rangle$ obeys $\tilde{C}_{0}(\omega)=1 / 4\left(\left\langle\tilde{z}(\omega) \tilde{z}^{*}(\omega)\right\rangle+\right.$ $\left.\left\langle\tilde{z}(-\omega) \tilde{z}^{*}(-\omega)\right\rangle\right)$ and thus has the form

$\tilde{C}_{0}(\omega) \simeq \frac{D_{\mathrm{eff}}}{K_{\mathrm{eff}}^{2}+\Lambda_{\mathrm{eff}}^{2}\left(\omega_{0}^{\mathrm{eff}}-\omega\right)^{2}}+\frac{D_{\mathrm{eff}}}{K_{\mathrm{eff}}^{2}+\Lambda_{\mathrm{eff}}^{2}\left(\omega_{0}^{\mathrm{eff}}+\omega\right)^{2}}$.

Provided that $\frac{1}{D_{\text {eff }}} \frac{\mathrm{d} D_{\text {eff }}}{\mathrm{d} \omega} \ll \frac{\Lambda_{\text {eff }}}{K_{\text {eff }}}$, we can neglect the dependence of $D_{\text {eff }}$ on $\omega$ and use the above expressions up to large $\omega_{0}^{\text {eff }}-\omega$. We then can estimate $\left\langle\rho^{2}\right\rangle=$ $\int(\mathrm{d} \omega / 2 \pi) \tilde{S}_{0}(\omega)$ to find

$$
\left\langle X^{2}\right\rangle=\frac{1}{2}\left\langle\rho^{2}\right\rangle \simeq \frac{D_{\mathrm{eff}}}{K_{\mathrm{eff}} \Lambda_{\mathrm{eff}}} .
$$

Vice versa, knowing the effective parameters (see below) and the second moment from the stationary density, we obtain from eq. (48) an estimate of the effective noise intensity.

\subsection{A simple case: $b^{\prime}=0$}

For $b^{\prime}=0$, we can use the quadratic approximation eq. (37) for $Q$ and derive the linear response $z_{1}$ for weak detuning as outlined in appendix E. In terms of the coefficients $a_{1,2}$ from eqs. (38), (39) we find

$$
z_{1}=\langle\rho Q\rangle \simeq \frac{\bar{f}}{2 d}\left[\left\langle\rho^{2}\right\rangle+i \delta \omega\left(a_{1}\left\langle\rho^{2}\right\rangle+a_{2}\left\langle\rho^{3}\right\rangle\right)\right]
$$

from which we can read off that $\alpha_{\text {eff }}=\alpha$ is not renormalized. Expanding the response function eq. (45) in small detuning, comparing to eq. (49), and using the expressions for $a_{1,2}$, eqs. (38), (39), we obtain

$$
\begin{aligned}
& K_{\mathrm{eff}}=\frac{2 d}{\left\langle\rho^{2}\right\rangle} \Lambda, \\
& \Lambda_{\mathrm{eff}} \simeq 2 \Lambda \\
& \times \frac{\langle\rho\rangle\left[\left\langle\rho^{3}\right\rangle\left\langle\rho^{4}\right\rangle-\left\langle\rho^{2}\right\rangle\left\langle\rho^{5}\right\rangle\right]+(d / b)\left\langle\rho^{2}\right\rangle\left[\left\langle\rho^{2}\right\rangle-\left\langle\rho^{3}\right\rangle\left\langle\rho^{-1}\right\rangle\right]}{\left\langle\rho^{2}\right\rangle^{2}\left[\left\langle\rho^{4}\right\rangle-\left\langle\rho^{5}\right\rangle\left\langle\rho^{-1}\right\rangle+(r / b)\left(\left\langle\rho^{2}\right\rangle-\left\langle\rho^{3}\right\rangle\left\langle\rho^{-1}\right\rangle\right)\right]} .
\end{aligned}
$$

The effective noise strength can be estimated for our simple example by combining (48) with (50), yielding

$$
D_{\mathrm{eff}} \simeq d \Lambda \Lambda_{\mathrm{eff}} .
$$

In the above equations the stationary moments

$$
\left\langle\rho^{n}\right\rangle=\int_{0}^{\infty} \mathrm{d} \rho \rho^{n+1} e^{-W(\rho) / d} / \int_{0}^{\infty} \mathrm{d} \rho \rho e^{-W(\rho) / d}
$$

can be expressed by error functions (even $n$ ) or Bessel functions (odd $n$ ); the resulting expression for $\Lambda_{\text {eff }}$ is lengthy and is therefore omitted here. It is, however, instructive to take a closer look at the explicit expression for the effective stiffness that can be written as follows:

$$
K_{\mathrm{eff}}=\frac{2 d b \Lambda I}{d-I r},
$$

where

$$
\begin{aligned}
I & =\int_{0}^{\infty} \mathrm{d} \rho \rho e^{-(r / 2 d) \rho^{2}-(b / 4 d) \rho^{4}} \\
& =\frac{(\pi d)^{1 / 2} e^{r^{2} /(4 d b)}}{2 b^{1 / 2}} \operatorname{erfc}\left(\frac{r}{2(d b)^{1 / 2}}\right),
\end{aligned}
$$

and erfc denotes the complementary error function. In particular, we find

$$
K_{\mathrm{eff}} \simeq \begin{cases}\Lambda r, & \text { for } r \rightarrow \infty \\ \Lambda(\pi b d)^{1 / 2}, & \text { for } r=0 \\ 2 \Lambda b d /|r|, & \text { for } r \rightarrow-\infty\end{cases}
$$

Please note that, in the presence of noise, the value of $K_{\text {eff }}$ according to eq. (50) is always positive, irrespective of the sign of $K=\Lambda r$. This implies that the Hopf bifurcation is concealed by the noise and the system behaves effectively like a stable fluctuating system, even for $r<0$. To illustrate this point, we discuss the weak-noise limit of the effective parameters in the oscillatory regime $(r<0)$. Here a saddle-point approximation of the moments in eq. (53) leads to

$$
\begin{aligned}
& K_{\mathrm{eff}} \simeq \frac{2 d b}{|r|} \Lambda \\
& \Lambda_{\mathrm{eff}} \simeq 16 \Lambda \frac{\left(2 r^{2}+d b\right) r^{2}\left(9 d b r^{2}+2 r^{4}+12 d^{2} b^{2}\right)}{\left(2 r^{2}+3 d b\right)^{2}\left(54 d b r^{2}+8 r^{4}+15 d^{2} b^{2}\right)}
\end{aligned}
$$

where the expression for $K_{\text {eff }}$ agrees with the $r \rightarrow-\infty$ limit in eq. (56). Adding noise to the limit cycle system prevents perfect phase locking and leads thereby to a linear response at weak forcing. The linear-response level at the best frequency is related to the noise level via $\chi\left(\omega=\omega_{0}\right) \sim 1 / K_{\text {eff }} \sim 1 / d$. In the limit of zero noise, the linear response diverges (as already discussed in sect. 2.2) because an arbitrary small signal leads to perfect phase entrainment with the external periodic stimulus.

We have determined the inverse of the real part and the slope of the imaginary part of $z_{1}$ at $\delta \omega=0$ integrating our numerical solution for $Q$ according to eq. (40). The resulting data for the two effective parameters $K_{\text {eff }}$ and $\Lambda_{\text {eff }}$ are compared to our exact result for $K_{\text {eff }}$ and our analytical approximation for $\Lambda_{\text {eff }}$ in fig. 4 as a function of noise intensity. Both curves show a very good agreement between numerics and analytical formulas for the full range of noise intensities. The weak-noise expressions eq. (57) and eq. (58) give also reasonable approximations for noise intensities up to $d=0.1$. The effective friction coefficient varies between 1 and 2 for our standard parameters.

A more direct verification of our simple approximation is provided by the spontaneous power spectrum and by the susceptibility of the normal form which amounts to setting $\Lambda=1$ and $\alpha=0$. Specifically, we look at spectrum and susceptibility of the real part $\operatorname{Re}(z)$ with respect to the signal $f(t)$ defined by $\chi=(1 / 2) \mathrm{d} z_{1} / \mathrm{d} \bar{f}$. Spectrum and susceptibility should be approximated by eq. (47) and eq. (45) with $K_{\text {eff }}, \Lambda_{\text {eff }}$ given by eq. (50) and eq. (51) with $\Lambda=1$ and $\alpha=0$. For both functions we 


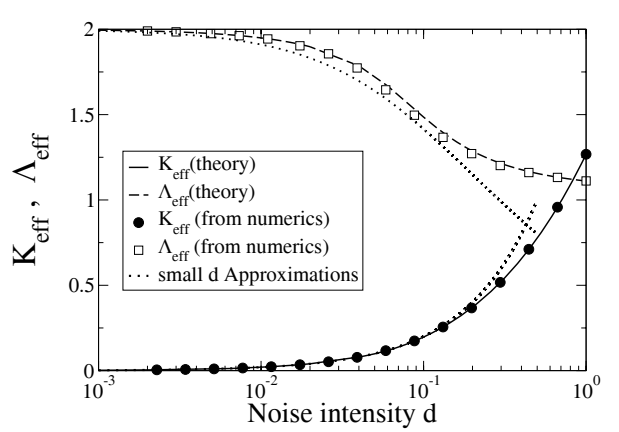

Fig. 4. The effective dimensionless parameters $K_{\text {eff }}$ and $\Lambda_{\text {eff }}$ on the oscillatory side of the bifurcation for $b^{\prime}=0$ and $\Lambda=1$ as determined from the full numerical solution (symbols), from the analytical results eq. (50) and eq. (51) (solid and dashed lines), and from the weak-noise expressions in eq. (57) and eq. (58) (dotted lines), shown as functions of the noise intensity $d$. Bare parameters are defined by eq. (D.3) with $\mathcal{B}=0$ (see appendix D).

A

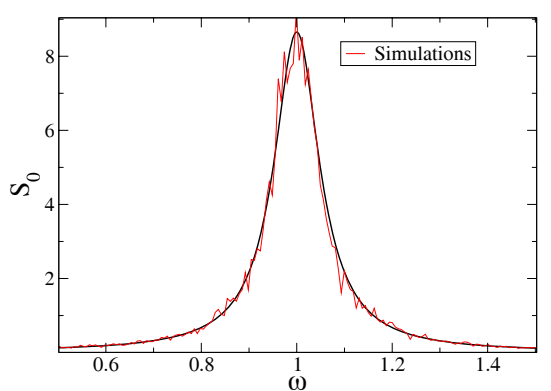

B

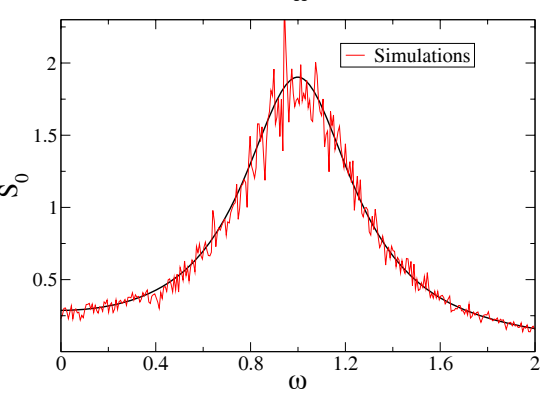

Fig. 5. The spontaneous power spectrum of $\operatorname{Re}(z)$ for $b^{\prime}=0$ and weak noise $(d=0.05)$ in (a) and moderate noise $(d=0.2)$ in (b). Theory is according to eq. (47) and eq. (48) both with $K_{\text {eff }}$ and $\Lambda_{\text {eff }}$ given in eqs. (50) and (51). Remaining parameters: $r=-1, b=1$.

find indeed a very good agreement between our approximation and simulation data as well as the numerical solution. For the power spectra at weak noise $(d=0.05)$ and moderate noise $(d=0.2)$ shown in fig. 5 we find that the Lorentzian shape describes well the spectral peak. It is expected that the small detuning expansion will work the better the sharper the peak is. In cases where the spectrum does not show a pronounced peak (i.e. at very strong noise) the approximation is expected to fail; in this limit, however, the normal form with phase-independent noise is most likely not appropriate anyway.

The susceptibility depicted in fig. 6 shows a similarly good agreement between the different numerical results

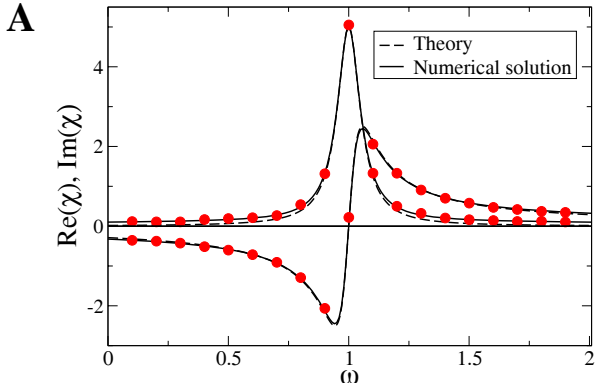

B

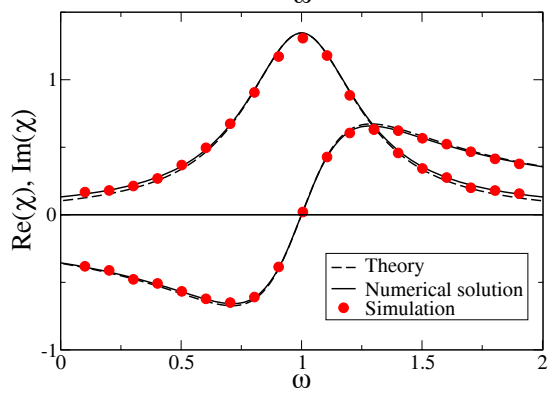

Fig. 6. The susceptibility of the real part with respect to a periodic stimulation of the normal form for $b^{\prime}=0$ and weak noise $(d=0.05)$ in (a) and moderate noise $(d=0.2)$ in (b). The solid line has been calculated from $\tilde{\chi}_{\operatorname{Re}(z), f}=\tilde{\chi}_{z, f} / 2=$ $z_{1} /(2 \bar{f})=\langle\rho Q\rangle /(2 \bar{f})$ using the numerical solution for $Q(\rho)$ for different driving frequencies $\omega$. The dashed line is the approximation eq. (45) using effective parameters according to eq. (50) and eq. (51) (with $\alpha=0, \Lambda=1$ ), and the symbols correspond to results of stochastic simulations of the normal form. Remaining parameters: $r=-1, b=1$.

(estimation by stochastic simulations and via the numerical solution for $Q$ ) and our approximation. Note that for the power spectrum other approximations have been derived (see $[5,6]$ and references therein) whereas we are not aware of any other analytical approach for the calculation of the susceptibility.

\subsection{General case: $\mathbf{b}^{\prime} \neq 0$}

Here we restrict ourselves to the numerical solution of the problem as follows. We determine the susceptibility for varying detuning $\omega_{0}-\omega$ and from its absolute value $\left|\tilde{\chi}_{z, f}\right|$ we find the effective eigenfrequency of the oscillator for $b^{\prime}>0$. From the numerical values of the real and imaginary parts of the susceptibility and their (numerically determined) derivatives with respect to $\omega$ we can extract the effective parameters $K_{\text {eff }}, \Lambda_{\text {eff }}, \alpha_{\text {eff }}$, and $\omega_{0}^{\text {eff }}$ which are shown in fig. 7 .

We also compare these data to the analytical results for $b^{\prime}=0$ in order to get an impression of the effect of a finite value of $b^{\prime}$. As can be seen, the dependence of $K_{\text {eff }}$ on $d$ is hardly changed; in general its value is slightly increased. Likewise, there are no drastic changes in $\Lambda_{\text {eff }}$ which now varies between 1 and 1.5. For a finite $b^{\prime}$, we observe a finite but moderate rescaling of $\alpha$ which lays between 0 and $\pi / 4$. Finally, the frequency $\omega_{0}^{\text {eff }}$ increases from a value of $\omega_{0}+\left(b^{\prime} / b\right)|r|=2$ to larger values. Such an 


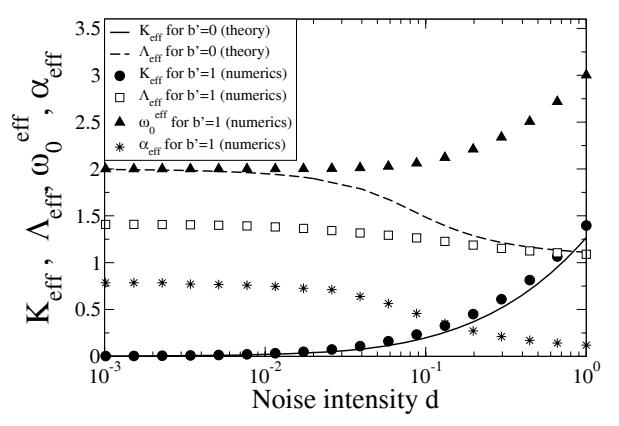

Fig. 7. The dimensionless effective parameters $K_{\text {eff }}, \Lambda_{\text {eff }}, \alpha_{\text {eff }}$ and $\omega_{0}^{\text {eff }}$ of the linear model for $b^{\prime}=1$ as determined from the full numerical solution as functions of the noise intensity $d$; for comparison we also show the analytical results eq. (50) and eq. (51) for $b^{\prime}=0$. Remaining parameters are defined by eq. (D.3) in appendix D.

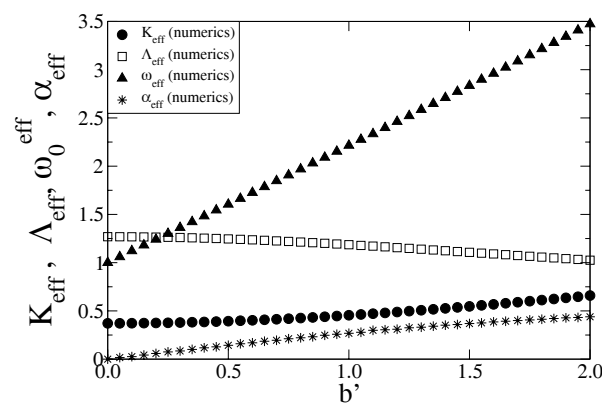

Fig. 8. The effective parameters $K_{\text {eff }}, \Lambda_{\text {eff }}, \alpha_{\text {eff }}$, and $\omega_{0}^{\text {eff }}$ of the effective model for $d=0.2$ as determined from the full numerical solution as functions of $b^{\prime}$. Remaining parameters are $r=-1, b=1, \Lambda=1$.

increase in the oscillation frequency with growing noise is typical for a nonlinear system (see, e.g., a few examples in $[23])$.

In fig. 8 we show the dependence of the effective parameters for a moderate noise $(d=0.2)$. Both $K_{\text {eff }}$ and $\Lambda_{\text {eff }}$ do not vary strongly with $b^{\prime}$, whereas $\omega_{0}^{\text {eff }}$ and $\alpha_{\text {eff }}$ increase monotonically with $b^{\prime}$.

\section{Discussion - relation to the hair bundle oscillator}

Because hair bundles can exhibit noisy spontaneous oscillations, they provide a unique experimental system to assay our theoretical predictions on the behavior of noisy oscillators. Using hair cells from the bullfrog's sacculus, both the autocorrelation function and the linear-response function of oscillatory hair bundles have been measured in vitro; these results as well as experimental details are published in ref. [18]. For a hair bundle oscillating at frequency $\omega_{0}^{\text {eff }} / 2 \pi \simeq 8 \mathrm{~Hz}$ with $\left\langle X^{2}\right\rangle \simeq 1.96 \cdot 10^{-16} \mathrm{~m}^{2}$, fitting the linear-response function to eq. (45) provided the estimates $K_{\text {eff }} \simeq 1.04 \cdot 10^{-4} \mathrm{~N} / \mathrm{m}, \Lambda_{\mathrm{eff}} \simeq 6.5 \cdot 10^{-6} \mathrm{~N} \mathrm{~s} / \mathrm{m}$ and $\alpha_{\mathrm{eff}} \simeq 0$.

The magnitude of the fluctuations $D_{\text {eff }} \simeq 1.4$. $10^{-25} \mathrm{~N}^{2} \mathrm{~s}$ was measured by fitting the spectral density of bundle movement to eq. (47). Using the approximation given by eq. (52), the noise strength can be estimated as $d \Lambda \simeq D_{\text {eff }} / \Lambda_{\text {eff }} \simeq 2.1 \cdot 10^{-20} \mathrm{Nm}$. Equivalently, we can use eq. (50) to find $d \Lambda=K_{\text {eff }}\left\langle X^{2}\right\rangle \simeq 2.03 \cdot 10^{-20} \mathrm{Nm}$. The noise strength $d \Lambda$ has units of energy and can be compared to $k_{B} T$. In this sense, it provides a definition of an effective temperature

$$
\bar{T}_{\mathrm{eff}}=d \Lambda / k_{B} \simeq \frac{D_{\mathrm{eff}}}{k_{B} \Lambda_{\mathrm{eff}}},
$$

which satisfies $(1 / 2) K_{\text {eff }}\left\langle X^{2}\right\rangle \simeq(1 / 2) k_{B} \bar{T}_{\text {eff }}$. Here, we find $d \Lambda \simeq 6 k_{B} T$, suggesting that the energy scale in the noise is six times stronger than that of thermal fluctuations of a passive system with the same stiffness. Note that these estimates are based on the assumption that the coefficient $B$ of the nonlinearity in eq. (14) is real. For moderate noise, however, the effective values of $K_{\text {eff }}$ and $\Lambda_{\text {eff }}$ show only weak variations upon varying the imaginary part $b^{\prime}$ of the bare parameter $B$ (fig. 8).

The response of oscillatory hair bundles to sinusoidal stimuli of increasing magnitudes has been previously measured [20]. Near the bundle's characteristic frequency of spontaneous oscillation and for sufficiently strong stimuli, the bundle's response displays a compressive nonlinearity. This behavior is similar to that of a deterministic system that operates close to a Hopf bifurcation. In the presence of noise, however, the bifurcation is concealed. A detailed description of the effects of noise on the nonlinear response of such active oscillatory system is lacking. Furthermore, higher-order nonlinearities in the dynamics might be present as, for instance, in the case of a subcritical Hopf bifurcation. In the future, we will extend the approach developed here to address these problems, which play an important role for signal detection by sensory systems.

We thank S. Camalet, T. Duke, and A.J. Hudspeth for stimulating collaborations.

\section{Appendix A. Linear transformation of the dynamic equations}

In order to put our system in the normal form, we first rewrite eqs. (1) and (2) in matrix form

$$
\dot{x}_{i}=A_{i j} x_{j}+f_{i},
$$

where the index $i=x, a$ denotes the two components. The variables are related to those in eqs. (1) and (2) by $x_{x}=X, x_{a}=F_{a}, f_{x}=F_{\text {ext }} / \lambda, f_{a}=0$. The matrix $A_{i j}$ is given by

$$
\mathbf{A}=\left(\begin{array}{cc}
-k / \lambda & 1 / \lambda \\
-\bar{k} / \beta & -1 / \beta
\end{array}\right) .
$$

Because the system we are describing is oscillating, the eigenvalues of the matrix $\mathbf{A}$ are complex conjugate. We 
denote them as $-r-i \omega_{0}$ and $-r+i \omega_{0}$, where

$$
\begin{aligned}
r & =\frac{1}{2}(k / \lambda+1 / \beta) \\
\omega_{0} & =\left(\bar{k} /(\lambda \beta)-\frac{1}{4}(k / \lambda-1 / \beta)^{2}\right)^{1 / 2} .
\end{aligned}
$$

We diagonalize the matrix A using the corresponding transformation matrix $\mathbf{M}$ :

$$
\mathbf{M}^{-1} \mathbf{A M}=\left(\begin{array}{lr}
-r-i \omega_{0} & 0 \\
0 & -r+i \omega_{0}
\end{array}\right) .
$$

This transformation matrix is given by

$$
\mathbf{M}=\frac{1}{2}\left(\begin{array}{cc}
1 & 1 \\
v & v^{*}
\end{array}\right)
$$

with

$$
\mathbf{M}^{-1}=\frac{2}{v^{*}-v}\left(\begin{array}{rr}
v^{*} & -1 \\
-v & 1
\end{array}\right)
$$

where

$$
v=\frac{1}{2}(k-\lambda / \beta)-i \lambda \omega_{0} .
$$

Defining the complex variables $z_{i}=M_{i j}^{-1} x_{j}$, the two components of $z_{i}$ are complex conjugate: $z=z_{x}=z_{a}^{*}$. The system can thus be described by the single complex equation

$$
\dot{z}=-\left(r+i \omega_{0}\right) z+f,
$$

where we have defined the complex force $f=M_{1 j}^{-1} f_{j}$. Note that with the choice of $\mathbf{M}$ given in eq. (A.6), $X=$ $\operatorname{Re}(z)$. The relation between the external force $F_{\text {ext }}$ and the complex force $f$ is given by

$$
f=\frac{e^{-i \alpha}}{\Lambda} F_{\text {ext }},
$$

where $\Lambda$ and $\alpha$ are, respectively, the amplitude and phase of

$$
\Lambda e^{i \alpha}=\lambda\left(1-\frac{i}{2 \omega_{0}}\left(\frac{k}{\lambda}-\frac{1}{\beta}\right)\right)^{-1} .
$$

\section{Appendix B. Normal form and Fourier modes}

The experimentally relevant variable $X$ is in general a nonlinear function of the complex variable $z$ whose dynamics is described by the normal form (eq. (14))

$$
\begin{aligned}
X= & \operatorname{Re}(z)+a_{1} z z^{*}+a_{2} \operatorname{Re}\left(z^{2}\right)+a_{3} \operatorname{Im}\left(z^{2}\right) \\
& +b_{1} \operatorname{Re}\left(z^{3}\right)+b_{2} \operatorname{Re}\left(z\left(z^{*}\right)^{2}\right) \\
& +b_{3} \operatorname{Im}\left(z^{3}\right)+b_{4} \operatorname{Im}\left(z\left(z^{*}\right)^{2}\right)
\end{aligned}
$$

in which we have limited the expansion to third order in $z$. The coefficients $a_{n}$ and $b_{n}$ depend on the model in question and ensure that the coordinate change eliminates all nonlinear terms from the dynamic equations but the generic terms of the normal form. Note that there is no term of the form $z^{2} z^{*}$ in eq. (B.1) [1].
We assume that the external stimulus is sinusoidal, $F_{\text {ext }}(t)=F_{1} e^{-i \omega t}+F_{-1} e^{i \omega t}$, with $F_{-1}=F_{1}^{*}$. The system is nonlinear and will thus respond with all higher harmonics. We write $X(t)=\sum_{n} X_{n} e^{-i n \omega t}$, in which each Fourier mode $X_{n}$ can be measured experimentally. It can been shown [10] that if the system operates near a Hopf bifurcation, the first Fourier mode dominates and obeys an expansion of the form

$$
F_{1}=A_{X F} X_{1}+B_{X F}\left|X_{1}\right|^{2} X_{1}+O\left(\left|X_{1}\right|^{4} X_{1}\right) .
$$

By using eq. (B.1) and the normal form eq. (14), we seek a relation between the coefficients $A_{X F}$ and $B_{X F}$ and the linear and nonlinear coefficients of the normal form. Writing $z=\sum_{n} z_{n} e^{-i n \omega t}$, we have

$$
\begin{aligned}
z^{3} & =\sum_{n k l} z_{n} z_{k} z_{l} e^{-i(n+k+l) \omega t}, \\
z\left(z^{*}\right)^{2} & =\sum_{n k l} z_{n} z_{k}^{*} z_{l}^{*} e^{-i(n-k-l) \omega t} .
\end{aligned}
$$

Using such expressions, we can express the Fourier modes $X_{n}$ of $X$ in terms of the $z_{n}$. For the $m$-th Fourier mode, we find

$$
\begin{aligned}
2 X_{m}= & z_{m}+z_{-m}+2 a_{1} \sum_{n} z_{m+n} z_{n}^{*} \\
& +a_{2} \sum_{n}\left(z_{m+n} z_{-n}+z_{m+n}^{*} z_{-n}^{*}\right) \\
& -i a_{3} \sum_{n}\left(z_{m+n} z_{-n}-z_{m+n}^{*} z_{-n}^{*}\right) \\
& +b_{1} \sum_{n k}\left(z_{m+n+k} z_{-n} z_{-k}+z_{m+n+k}^{*} z_{-n}^{*} z_{-k}^{*}\right) \\
& +b_{2} \sum_{n k}\left(z_{m+n+k} z_{n}^{*} z_{k}^{*}+z_{m+n+k}^{*} z_{n} z_{k}\right) \\
& -i b_{3} \sum_{n k}\left(z_{m+n+k} z_{-n} z_{-k}-z_{m+n+k}^{*} z_{-n}^{*} z_{-k}^{*}\right) \\
& -i b_{4} \sum_{n k}\left(z_{m+n+k} z_{n}^{*} z_{k}^{*}-z_{m+n+k}^{*} z_{n} z_{k}\right) .
\end{aligned}
$$

Knowing the modes $z_{k}$, we can thus discuss the modes $X_{k}$.

For simplicity, we assume that the component $F_{-1}$ of the stimulus, which corresponds to the frequency $-\omega$, can be neglected. This approximation is valid as long as we focus on the response of the system to frequencies $\omega \simeq \omega_{0}$ close to resonance and as the system operates near the bifurcation. This implies $\left|\omega_{0}\right| \gg|r|$. In this case, because the component $F_{-1}$ stimulates the system far from resonance at $\omega \simeq-\omega_{0}$, it will not affect significantly the active, resonant response elicited by the component $F_{1}$ at $\omega \simeq \omega_{0}$. We therefore write $f(t)=e^{-i(\alpha+\omega t)} F_{1} / \Lambda$ and therefore

$$
f_{1}=e^{-i \alpha} F_{1} / \Lambda \text {. }
$$

Using (14), we find in this simple case that all modes $z_{n}=$ 0 vanish except for $z_{1}$ which obeys

$$
f_{1}=A(\omega) z_{1}+B\left|z_{1}\right|^{2} z_{1}+O\left(\left|z_{1}\right|^{4} z_{1}\right),
$$


where $A(\omega)=i\left(\omega_{0}-\omega\right)+r$. Although only the first Fourier modes is nonzero for the complex variable $z$, eq. (B.4) generates all Fourier coefficients $X_{k}$ for the variable $X$, with $X_{-k}=X_{k}^{*}$. Using eq. (B.4) with $m=1$, we find

$$
\begin{aligned}
F_{1} & =A_{X F} X_{1}+B_{X F}\left|X_{1}\right|^{2} X_{1}+O\left(\left|X_{1}\right|^{4} X_{1}\right) \\
& =A_{X F} z_{1} / 2+B_{X F}\left|z_{1}\right|^{2} z_{1} / 8+O\left(\left|z_{1}\right|^{4} z_{1}\right) \\
& =\Lambda e^{i \alpha}\left(A z_{1}+B\left|z_{1}\right|^{2} z_{1}\right)+O\left(\left|z_{1}\right|^{4} z_{1}\right) .
\end{aligned}
$$

Note that, because the nonlinear terms in eq. (B.4) are not of the form $|z|^{2} z$, they do not contribute to the cubic nonlinearity in eq. (B.7). We thus find that the observed linear and nonlinear coefficients for the response of $X_{1}$ are simply related to the coefficients of the normal form for $z$ given by eq. (17). Because, as stated above we neglected the contribution of $F_{-1}$ and thus $z_{-1}$, these relations are only approximations.

\section{Appendix C. Phase-dependent noise}

In the presence of noise, eq. (3) becomes

$$
\dot{x}_{i}=A_{i j} x_{j}+f_{i}+\eta_{i},
$$

in which $\eta_{i}(t)$ are random forces acting on the hair bundle and on the motors [21]. We assume white noise with correlations $\left\langle\eta_{i}(t) \eta_{j}\left(t^{\prime}\right)\right\rangle=2 d_{i} \delta_{i j} \delta\left(t-t^{\prime}\right)$ and strength $d_{i}$. In eq. (20) of the normal form of a noisy oscillator, the noise $\xi=M_{1 j}^{-1} \eta_{j}$ is then given by

$$
\xi(t)=\frac{i}{\lambda \omega_{0}}\left(\eta_{2}(t)-v^{*} \eta_{1}(t)\right)
$$

The noise correlations are of the form $\left\langle\xi(t) \xi^{*}\left(t^{\prime}\right)\right\rangle=$ $4 D \delta\left(t-t^{\prime}\right)$ and $\left\langle\xi(t) \xi\left(t^{\prime}\right)\right\rangle=4 D^{\prime} \delta\left(t-t^{\prime}\right)$, with

$$
\begin{aligned}
D & =\frac{1}{2 \omega^{2} \lambda^{2}}\left(|v|^{2} d_{1}+d_{2}\right), \\
D^{\prime} & =-\frac{1}{2 \omega^{2} \lambda^{2}}\left(\left(v^{*}\right)^{2} d_{1}+d_{2}\right) .
\end{aligned}
$$

Note that the phase-dependent amplitude of the noise $\left|D^{\prime}\right|$ is in general of a similar magnitude as $D$. The autocorrelation function $\tilde{C}_{0}(\omega)=\langle\tilde{X}(\omega) \tilde{X}(-\omega)\rangle$ is given by

$$
\begin{aligned}
\tilde{C}_{0}= & 1 / 4\left(\left\langle\tilde{z}(\omega) \tilde{z}^{*}(\omega)\right\rangle+\left\langle\tilde{z}(-\omega) \tilde{z}^{*}(-\omega)\right\rangle\right. \\
& \left.+\langle\tilde{z}(\omega) \tilde{z}(-\omega)\rangle+\left\langle\tilde{z}^{*}(\omega) \tilde{z}^{*}(-\omega)\right\rangle\right) .
\end{aligned}
$$

Because $\tilde{z} \simeq \tilde{\chi}_{z F} \tilde{\xi}$, we find that the phase-dependent contributions to $\tilde{C}_{0}$ at $\omega=\omega_{0}$ are of the order

$$
\left\langle\tilde{z}\left(\omega_{0}\right) \tilde{z}\left(-\omega_{0}\right)\right\rangle \simeq \frac{4 D^{\prime}}{K^{2}+2 i K \Lambda \omega_{0}} .
$$

This has to be compared to the phase-invariant contribution

$$
\left\langle\tilde{z}\left(\omega_{0}\right) \tilde{z}^{*}\left(\omega_{0}\right)\right\rangle \simeq \frac{4 D}{K^{2}} .
$$

Because $\left|D^{\prime}\right|$ is of the same oder of magnitude as $D$, the contribution of phase-dependent noise to $\tilde{C}_{0}$ can be neglected if $K \ll \Lambda \omega_{0}$. This is the case when the autocorrelation is sharply peaked, i.e. near the bifurcation for sufficiently weak noise.

\section{Appendix D. Dimensionless expression of the normal form}

A dynamical system that operates near a Hopf bifurcation can be transformed into the normal form eq. (14) by a sequence of analytic, but nonlinear, coordinate changes (see chapt. 2.2 in ref. [1]). The normal form can be further transformed by $z=\bar{z} e^{-i \omega t}$ into

$$
\dot{\bar{z}}=-\left(r+i\left[\omega_{0}-\omega\right]\right) \bar{z}-B|\bar{z}|^{2} \bar{z}+\bar{f}+\bar{\xi}(t),
$$

where $\bar{\xi}$ is a white Gaussian noise which possesses the same statistics as $\xi(t)$ given in eq. (14).

By setting $\bar{t}=|r| t$ and $\hat{\bar{z}}=\sqrt{\frac{b}{|r|}} \bar{z}$ we can further transform eq. (D.1) into

$$
\begin{aligned}
\dot{\bar{z}}= & \left(i \frac{\omega-\omega_{0}}{|r|}-\frac{r}{|r|}\right) \hat{\bar{z}}-\left(1+i \frac{b^{\prime}}{b}\right)|\hat{\bar{z}}|^{2} \hat{\bar{z}} \\
& +\sqrt{\frac{b}{|r|^{3}}} \bar{f}+\frac{\sqrt{b}}{|r|} \bar{\xi}(\bar{t}) .
\end{aligned}
$$

Assuming $r<0$ and denoting

$$
\Omega=\frac{\omega_{0}-\omega}{|r|}, \quad \mathcal{B}=\frac{b^{\prime}}{b}, \quad \overline{\bar{f}}=\sqrt{\frac{b}{|r|^{3}}} \bar{f}, \quad \bar{d}=\frac{b}{r^{2}} d
$$

we can thus write the dimensionless equation

$$
\dot{\overline{\bar{z}}}=(1-i \Omega) \hat{\bar{z}}-(1+i \mathcal{B})|\hat{\bar{z}}|^{2} \hat{\bar{z}}+\overline{\bar{f}}+\overline{\bar{\xi}}(t),
$$

where the white noise has the intensity $\bar{d}$, i.e. $\left\langle\overline{\bar{\xi}}_{i}(t) \overline{\bar{\xi}}_{j}\left(t^{\prime}\right)\right\rangle=$ $2 \bar{d} \delta_{i, j} \delta\left(t-t^{\prime}\right)$. With these transformations, we have thus reduced the number of parameters $\left(r, b, \omega_{0}\right)$. For the numerical evaluations, we have considered $b=1, r=-1$ and $\omega_{0}=1$ which reflects the discussed parameter redundancy. Note that this rescaling changes both the magnitude of the driving and the noise intensity.

\section{Appendix E. Small-detuning approximation for $\mathbf{b}^{\prime}=0$}

The approximations for $Q(z)$ and for the effective parameter $\Lambda_{\text {eff }}$ at small detuning can be obtained as follows. We multiply the differential equation eq. (30) governing $Q(\rho)$ by $\rho^{k} P_{s}(\rho)$ with $k=0,1,2, \ldots$ and integrate over $\rho$. This yields the following equation relating certain moments of the perturbation with moments of the unperturbed system:

$$
\begin{aligned}
& D\left[1-k^{2}\right]\left\langle\rho^{k-2} Q\right\rangle+[k r-i \delta \omega]\left\langle\rho^{k} Q\right\rangle+b k\left\langle\rho^{k+2} Q\right\rangle= \\
& \frac{\bar{f}}{2 d}\left(r\left\langle\rho^{k+1}\right\rangle+b\left\langle\rho^{k+3}\right\rangle\right) .
\end{aligned}
$$

For varying integer $n$, this represents an infinite hierarchy of moment equations that cannot be solved exactly for all moments. We recall that the linear response is given by 
$z_{1}=\langle\rho Q\rangle$. For small values of $\delta \omega$ we may expand $Q$ with respect to the detuning

$$
Q=Q_{0}+i \delta \omega Q_{1}
$$

where both functions $Q_{0}, Q_{1}(\rho)$ are real and the first one corresponds to the solvable case $\omega=\omega_{0}$, i.e. $Q_{0}=$ $\bar{f} \rho /(2 d)$. For the moments involving $Q_{1}$ we obtain for $k=0$ and $k=1$

$$
\begin{aligned}
D\left\langle\rho^{-2} Q_{1}\right\rangle & =-\left\langle Q_{0}\right\rangle, \\
r\left\langle\rho Q_{1}\right\rangle+b\left\langle\rho^{3} Q_{1}\right\rangle & =-\left\langle\rho Q_{0}\right\rangle .
\end{aligned}
$$

The first equation represents an exact solution for $\left\langle\rho^{-2} Q_{1}\right\rangle$ which is, unfortunately, the only moment that can be exactly calculated. Approximating the function $Q_{1}(\rho)=$ $\bar{f} /(2 d)\left[a_{1} \rho+a_{2} \rho^{2}\right]$, we can choose $a_{1}$ and $a_{2}$ such that these two relations are fulfilled. Inserting the quadratic ansatz in eq. (E.3), we obtain two linear equations in $a_{1}$ and $a_{2}$ with coefficients proportional to certain moments of the unperturbed system. Their solution is given in eq. (38) and eq. (39).

\section{References}

1. S. Wiggins, Introduction to Applied Nonlinear Dynamical Systems and Chaos (Springer, New York, 1990).

2. R.D. Hempstead, M. Lax, Phys. Rev. 161, 350 (1967).

3. R.L. Stratonovich, Topics in the Theory of Random Noise (Gordon and Breach, New York, 1967).

4. H. Risken, The Fokker-Planck Equation (Springer, Berlin, 1984).
5. O.V. Ushakov, H.-J. Wünsche, F. Henneberger, I.A. Khovanov, L. Schimansky-Geier, M.A. Zaks, Phys. Rev. Lett. 95, 123903 (2005).

6. J.P. Gleeson, F. O'Doherty, SIAM J. Appl. Math. 66, 1669 (2006).

7. C. Meunier, A.D. Verga, J. Stat. Phys. 50, 345 (1988).

8. K. Wiesenfeld, J. Stat. Phys. 38, 1071 (1985).

9. Y. Choe, M.O. Magnasco, A.J. Hudspeth, Proc. Natl. Acad. Sci. U.S.A. 95, 15321 (1998).

10. S. Camalet, T. Duke, F. Jülicher, J. Prost, Proc. Natl. Acad. Sci. U.S.A. 97, 3183 (2000).

11. V.M. Eguiluz, M. Ospeck, Y. Choe, A.J. Hudspeth, M.O. Magnasco, Phys. Rev. Lett. 84, 5232 (2000).

12. T.A.J. Duke, F. Jülicher, Active Processes and Otoacoustic Elements, edited by G.A. Manley, R.R. Fay, A.N. Popper, Springer Handb. Audit. Res., Vol. 30 (Springer, New York, 2008).

13. A.C. Crawford, R. Fettiplace, J. Physiol. 364, 359 (1985).

14. J. Howard, A.J. Hudspeth, Proc. Natl. Acad. Sci. U.S.A. 84, 3064 (1987).

15. W. Denk, W.W. Webb, Hear. Res. 60, 89 (1992).

16. P. Martin, A.J. Hudspeth, Proc. Natl. Acad. Sci. U.S.A. 96, 14306 (1999).

17. P. Martin, A.D. Mehta, A.J. Hudspeth, Proc. Natl. Acad. Sci. U.S.A. 97, 12026 (2000).

18. P. Martin, A.J. Hudspeth, F. Jülicher, Proc. Natl. Acad. Sci. U.S.A. 98, 14380 (2001).

19. P. Martin, D. Bozovic, Y. Choe, A.J. Hudspeth, J. Neurosci. 23, 4533 (2003).

20. P. Martin, A.J. Hudspeth, Proc. Natl. Acad. Sci. U.S.A. 98, 14386 (2001).

21. B. Nadrowski, P. Martin, F. Jülicher. Proc. Natl. Acad. Sci. U.S.A. 101, 12195 (2004).

22. D. Clausznitzer, B. Lindner, F. Jülicher, P. Martin, Phys. Rev. E 77, 041901 (2008).

23. B. Lindner, J. García-Ojalvo, A. Neiman, L. SchimanskyGeier, Phys. Rep. 392, 321 (2004). 